\title{
Spatial-temporal peculiarities of periodical PILS in ferroelectric PR-crystals
}

\author{
A.N. Morozovska, V.V. Obukhovsky \\ Taras Shevchenko Kyiv University, Radiophysic Department, 64 Volodymyrs'ka str., 01033 Kyiv, Ukraine \\ e-mail:morozo@mail.i.com.ua,obukhovsky@univ.kiev.ua
}

\begin{abstract}
In the paper we theoretically consider the dynamics of the inner field and the spatial-temporal features of periodical photoinduced light scattering in photo-ferroelectrics caused by stationary laser illumination. Not only the longitudinal but also the transverse photovoltaic current and the diffusion mechanism of carrier transfer are taken into account. It has been shown that, under the definite relation between the material parameters, among all possible scenarios of transverse instabilities the boundary circle will be realized in the system. Due to this phenomenon the periodical photoinduced light scattering appears. So the great attention has been paid to the description of the optical autowaves generation in pseudoilmenites. All the main theoretical results are in a good agreement with the available experimental data on periodical photoinduced light scattering in $\mathrm{LiNbO}_{3}: \mathrm{Fe}$.
\end{abstract}

Keywords: photorefractive crystals, transverse photovoltaic current, optical autowaves.

Paper received 25.05.03; accepted for publication 17.06.03.

\section{Introduction}

This work is devoted to the theoretical description of autowave (i.e. periodical) photoinduced light scattering (PILS) in photorefractive crystals (PRC). This phenomenon was observed in $\mathrm{LiNbO}_{3}: \mathrm{Fe}$ (with weight concentration of impurity $0.03 \%$ ) by authors [1] more then 10 years ago. The phenomenon of autowave PILS consists in the following:

During pumping along the optical axis (regime of the photoinduced blenching) by a cylindrical light beam (diameter $d=3 \mathrm{~mm}, \lambda_{0}=0.44 \mu \mathrm{m}, P_{L}=20 \mathrm{~mW}$ ) focused onto the surface of the crystal by a lens, we observed a periodic appearance of a scattering in the form of a cone directed opposite the pump. On a screen the autowaves are seen as rings, which spread out from a common centre (Fig. 1).

The previous attempts to describe this phenomenon within the framework of the theory of recharging waves [2]-[4] appeared not very successful. The approach developed in Ref. [5-8], in spite of its indisputable scientific significance, failed to be applied directly to the autowave PILS. Namely the spatial indicatrix and temporal spectrum of the autowave PILS were neither obtained nor compared with the experiment. Also the quali- tatively correct generation conditions and the simple physical explanation of this phenomenon mechanism have been absent.

During the previous three years we succeed to develop the theory free of the aforementioned disadvantages [9-11]. The marked degree of this success is obliged to the direct access to the experiment, i.e. the autowave PILS in $\mathrm{LiNbO}_{3}: \mathrm{Fe}$ was observed in our lab for the first time and have been actively studied since. That is why all the basic hypotheses were proved with the help of the directly carried out experiments. In consequence of this fact the adequate physical and mathematical description of the phenomenon have been proposed. Our description has the following distinctive features.

1. The sources of both the backward scattering and the internal field are the volume inhomogeneity of the refractive index, the photoactive impurity concentration and so on.

2. For the autowave scattering existence it is necessary not only the longitudinal field but also the transversal one. The latter field exists only in PRC with nonzero $\beta_{\perp}$, i.e. with transversal PV-current. Diffusion in the longitudinal direction is also essential.

3. The backward PILS is amplified for the definite crystal orientation. In this case autowaves could exist. 
A.N. Morozovska, V.V. Obukhovsky: Spatial-temporal peculiarities of periodical PILS ...

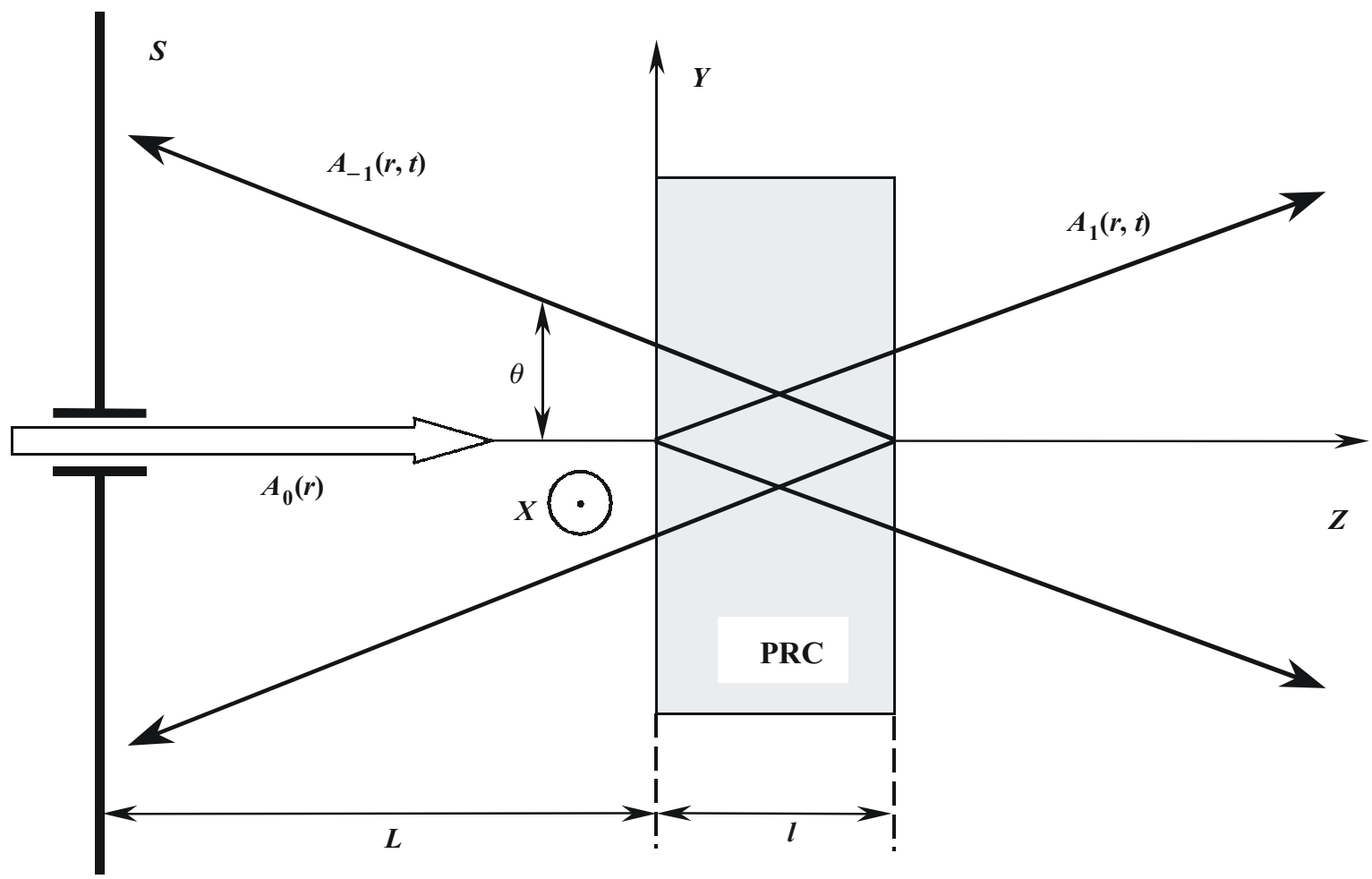

Fig. 1. Scheme of the AW PILS investigation.

4. The pump wave due to the electro-optical (EO) effect creates the gaussian lens near the front crystal plane and focuses on this lens. Therefore the focusing of the pump wave is extremely important.

5. The space distribution of dielectric permittivity and, therefore, the scattering radiation has the radial symmetry in the crystals of $\mathrm{C}_{3 \mathrm{~V}}$ point group. Only for this symmetry group the instability with the fixed wave vector absolute value and all possible directions is realized in the system.

Actually we propose the sufficiently exact analytical description of the dynamical, in particular periodical, PILS in PRC. This description allows one to classify photorefractive (PR) media, in which this type of phenomena can be observed. Also we carried out the detailed comparison with the available experimental data and demonstrate both the quantitative and full qualitative agreement with it.

\section{General equations and hypothesis}

\subsection{The problem}

Let us consider the photoinduced effects in PRC without inversion center. Namely we are interested in the generation threshold and evolution of the electric fields transverse instability in PRC under steady state laser irradiation along polar (optical) axis.
The geometry of our calculations is the following. The pump wave vector is directed along crystal polar axis Z, pump polarization $\vec{e}_{1}$ lies in XY-plain, coordinate origin is in the pump beam center in the front plain of the crystal with symmetry $\mathrm{C}_{3 \mathrm{~V}}$ and definite thickness $\ell$. The sample can be rega $=$ rded infinite in transversal direction, because laser beam diameter is much less than the transverse size of the sample (see Fig. 1).

Laser field in PRC in the smooth amplitudes' approximation has the view:

$\vec{E}^{\omega}=\vec{A}_{1}(\vec{r}, t) \exp \left[i\left(k_{0} z-\omega t\right)\right]+$

$+\vec{A}_{-1}(\vec{r}, t) \exp \left[-i\left(k_{0} z+\omega t\right)\right]+c . c$.

Here $k_{0}=\omega \sqrt{\varepsilon_{0}} / c$ is the wave vector. The inner photoinduced electric field in the short-circuited sample has the following form:

$\vec{E}^{f}=\vec{E}_{s}^{f}+\vec{U}^{f} \exp \left[2 i k_{0} z\right]+c . c$.

$\int_{-\ell}^{\ell} \vec{E}^{f}=0 \Rightarrow \vec{E}_{s}^{f} \approx 0$

\subsection{The system of equations for the laser field}

The equations for the laser fields' smooth amplitudes owing to the linear electro-optical effect are the following: 


$$
\left\{\begin{aligned}
\frac{\partial \vec{A}_{1}}{\partial z}-\left(\frac{i}{2 k_{0}}\right) \Delta_{\perp} \vec{A}_{1}+\eta \vec{A}_{1}= \\
=i \frac{k_{0}}{2}\left[(\Delta \widehat{\varepsilon}+\delta \varepsilon(\vec{r})) \vec{A}_{-1}+\Delta \varepsilon_{P R} \vec{A}_{1}\right] \\
\\
\quad \frac{\partial A_{-1}}{\partial z}+\left(\frac{i}{2 k_{0}}\right) \Delta_{\perp} \vec{A}_{-1}-\eta \vec{A}_{-1}= \\
=-i \frac{k_{0}}{2}\left[\left(\Delta \hat{\varepsilon}^{*}+\delta \varepsilon^{*}(\vec{r})\right) \vec{A}_{1}+\Delta \varepsilon_{P R} \vec{A}_{-1}\right]
\end{aligned}\right.
$$

Here $\eta$ is the optical absorption coefficient. $\varepsilon=$ $=\varepsilon_{0}\left(1+\Delta \vec{\varepsilon}(\vec{r}, t)+\delta \varepsilon(\vec{r})+\Delta \varepsilon_{P R}\right)$ is the dielectric permittivity, where the relative variation $\Delta \widehat{\varepsilon}=\bar{\chi} \vec{U}^{f}$ corresponds to the wave vector $2 k_{0}, \bar{\chi}$ is electro-optic tensor, $\delta \varepsilon(\vec{r})$ is the bulk static fluctuations of dielectric permittivity with $2 k_{0}$ space period, $\Delta \varepsilon_{P R}$ is dielectric permittivity variation owing to the other photo-refraction mechanisms (namely absorption).

Let us assume, that $\vec{A}_{ \pm 1}=\vec{e}_{1} A_{ \pm 1}(\vec{r}, t)$, i.e. the pump polarization maintains, then one could obtain

$$
\frac{\partial A_{1}}{\partial z}-\left(\frac{i}{2 k_{0}}\right) \Delta_{\perp} A_{1}=i \frac{k_{0}}{2}\left[(\Delta \hat{\varepsilon}+\delta \varepsilon(\vec{r})) A_{-1}+\Delta \varepsilon_{P R} A_{1}\right],
$$

$$
\begin{aligned}
& \frac{\partial A_{-1}}{\partial z}+\left(\frac{i}{2 k_{0}}\right) \Delta_{\perp} A_{-1}= \\
& =-i \frac{k_{0}}{2}\left[\left(\Delta \hat{\varepsilon}^{*}+\delta \varepsilon^{*}(\vec{r})\right) A_{1}+\Delta \varepsilon_{P R} A_{-1}\right],
\end{aligned}
$$

where $\Delta \widehat{\varepsilon}=\vec{e}_{1}\left(\hat{\chi} \vec{U}^{f}\right) \vec{e}_{1}$. Note, that by definition $\vec{U}^{f}=\left(U_{z}^{f} \vec{e}_{z}+U_{\perp}^{f} \vec{e}_{\perp}\right)$, let us introduce $\Delta \varepsilon=\Delta \varepsilon_{\|}+\Delta \varepsilon_{\perp}$, where $\left\{\begin{array}{l}\Delta \varepsilon_{\|}=e_{1 i} e_{1 j} \chi_{i j, z} U_{z}^{f}, \\ \Delta \varepsilon_{\perp}=e_{1 i} e_{1 j} \chi_{i j, \perp} U_{\perp}^{f}\end{array}\right.$

Hereinafter $I$ is laser beam intensity, i.e.

$$
I=\left|A_{1}\right|^{2}+\left|A_{-1}\right|^{2}+A_{1} A_{-1}^{*} \exp \left[2 i k_{0} z\right]+c . c .
$$

\subsection{The system of equations for inner field}

If the acceptor levels are rather deep and completely filled, i.e. $\rho=e\left(n_{d}^{+}-n_{e}-n_{a}\right)$, Maxwell equation has the form: $\operatorname{div}\left(\widehat{\varepsilon}^{f} \vec{E}^{f}\right)=4 \pi e\left(n_{d}^{+}-n_{e}-n_{a}\right)$.

Charge conservation law must supplement eq. (7):

$$
\begin{aligned}
& \operatorname{div}\left[e \widehat{\mu} n_{e} \vec{E}^{f}+e D_{0} \nabla n_{e}+\frac{\hat{\varepsilon}^{f}}{4 \pi} \frac{\partial \vec{E}^{f}}{\partial t}\right]= \\
& =-\operatorname{div}\left[I\left(n_{d}-n_{d}^{+}\right) \hat{\beta}: \vec{e}_{1} \cdot \vec{e}_{1}^{*}\right] .
\end{aligned}
$$

Material equations for ionized donors' concentration is the following [12]:

$$
\frac{\partial n_{d}^{+}}{\partial t}=-\gamma_{R} n_{e} n_{d}^{+}+\gamma_{p} I\left(n_{d}-n_{d}^{+}\right)+\gamma_{T}\left(n_{d}-n_{d}^{+}\right) .
$$

In (7)-(9) the following designations are introduced: $n_{e}$ is the concentration of free electrons, $n_{d}$ is the concentration of donors, $n_{d}^{+}$is the concentration of ionized donors (traps), $n_{a}$ is the concentration of acceptors $\gamma_{R}$ is the capture coefficient of electron by trap, $\gamma_{p}$ is the photoionization coefficient, $\gamma_{T}$ is the thermalization coefficient, $e$ is the absolute value of the electron charge, $\varepsilon^{f}$ is static dielectric permittivity, $\mu$ is the electron mobility, $D_{0}$ is the diffusion coefficient, $\widehat{\beta}$ is the photovoltaic tensor. The quantities $\vec{E}^{f}, n_{d}^{+}, n_{e}$ are unknown.

\subsection{Approximations}

1. Adiabatic approximation for ionized donors evolution

$\gamma_{R} n_{e} n_{d}^{+}=\gamma_{p} I\left(n_{d}-n_{d}^{+}\right)+\gamma_{T}\left(n_{d}-n_{d}^{+}\right)$

2. The expansions similar to (2) is valid:

$n_{e}=n_{e}^{s}+\tilde{n}_{e} \exp \left[2 i k_{0} z\right]+c . c .$,

$n_{d}^{+}=n_{d s}^{+}+\tilde{n}_{d}^{+} \exp \left[2 i k_{0} z\right]+$ c.c.,

i.e. the harmonic $2 k_{0}$ is extracted from the rather broad fluctuations spectrum. It is caused by $A_{-1}^{*}$ and $\vec{U}^{f}$ in self-consistent manner.

3 . The smooth amplitudes approach assumes the following condition for the short-circuited sample:

$\operatorname{div}\left(\widehat{\varepsilon}^{f} \vec{E}^{f}\right)=2 i k_{0} \varepsilon_{z}^{f}\left(U_{z}^{f} \exp \left[2 i k_{0} z\right]-c . c.\right)=$ $=4 \pi e\left(\tilde{n}_{d}^{+}-\tilde{n}_{e}\right) \exp \left[2 i k_{0} z\right]+c . c$.

Also $n_{d s}^{+}=n_{e}^{s}+n_{a}$ owing to the acceptors.

4. Let us introduce the smooth function

$I_{s}=\left|A_{1}\right|^{2}+\left|A_{-1}\right|^{2}$.

The concentration $n_{e}^{s}=n_{d s}^{+}-n_{a}$ could be found from the quadratic equation:

$\gamma_{R}\left(n_{e}^{s}\right)^{2}+\left(\gamma_{p} I_{s}+\gamma_{T}+\gamma_{R} n_{a}\right) n_{e}^{s}-$

$-\left(\gamma_{p} I_{s}+\gamma_{T}\right)\left(n_{d}^{0}-n_{a}\right)=0$, 


\section{A.N. Morozovska, V.V. Obukhovsky: Spatial-temporal peculiarities of periodical PILS ...}

namely at the linear region of photo-ionization, i.e.

$\left(\gamma_{p} I_{s}+\gamma_{T}\right)<<\gamma_{R} n_{a}$, one obtains

$n_{e}^{s} \approx \frac{\gamma_{p} I_{s}+\gamma_{T}}{\gamma_{p} I_{s}+\gamma_{T}+\gamma_{R} n_{a}}\left(n_{d}^{0}-n_{a}\right)$,

$n_{d s}^{+} \approx n_{a}+\frac{\gamma_{p} I_{s}+\gamma_{T}}{\gamma_{p} I_{s}+\gamma_{T}+\gamma_{R} n_{a}}\left(n_{d}^{0}-n_{a}\right)$

Hereinafter it is convenient to introduce the designation for conductivity tensor:

$\hat{\sigma}\left(I_{s}\right)=e \widehat{\mu} n_{e}^{s}$.

The equations for $\tilde{n}_{e}, \tilde{n}_{d}^{+}, \vec{U}^{f}$ could be transformed as following.

$\tilde{n}_{d}^{+}=\tilde{n}_{e}+\frac{i k_{0} \varepsilon_{z}^{f}}{2 \pi e} U_{z}^{f}$,

$-\gamma_{R} n_{e}^{s}\left(\tilde{n}_{d}^{+}+\tilde{n}_{e}\right)-\left(\gamma_{p} I_{s}+\gamma_{T}\right) \tilde{n}_{d}^{+}+$

$+\gamma_{p}\left(n_{d}^{0}-n_{d s}^{+}\right) A_{1} A_{-1}^{*}=0$,

$\frac{\hat{\varepsilon}^{f}}{4 \pi} \frac{\partial}{\partial t} \vec{U}^{f}+\hat{\sigma} \vec{U}^{f}+e D_{0} 2 i k_{0} e_{z} \tilde{n}_{e}=$

$=-\widehat{\beta}: e_{1} \cdot \vec{e}_{1}^{*}\left(-\tilde{n}_{d}^{+} I_{s}+\left(n_{d}^{0}-n_{d s}^{+}\right) A_{1} A_{-1}^{*}\right)$.

The values $\tilde{n}_{e}, \tilde{n}_{d}^{+}$can be expressed in terms of $A_{i}, U_{z}^{f}$ from (17) and (18). Thus the following equation for the inner field could be derived from (19):

$$
\begin{aligned}
& \frac{\hat{\varepsilon}^{f}}{4 \pi} \frac{\partial}{\partial t} \vec{U}^{f}+\hat{\sigma}^{f} \vec{U}^{f}= \\
& =-\left(Q^{\prime} A_{1} A_{-1}^{*}-i \delta U_{z}^{f}\right) \cdot \widehat{\beta}: \vec{e}_{1} \cdot \vec{e}_{1}^{*}-i \vec{e}_{z}\left(D^{\prime} A_{1} A_{-1}^{*}\right) .
\end{aligned}
$$

2.5. The bacis system of equations for periodic PILS in PRC with $C_{3 m}$ symmetry

When using the definite convolutions of photovoltaic and electro-optic tensors (5) with pump beam polarization $\vec{e}_{1}=\left(e_{i x}, e_{i y}\right)$ for the crystal with $C_{3 m}$ symmetry [13]-[15], one could obtain the equations for $\Delta \varepsilon_{\|}=\Delta \varepsilon_{z}$, $\Delta \varepsilon_{\perp}=\Delta \varepsilon_{x}+\Delta \varepsilon_{y}$, instead of the ones for $U_{z}^{f}, U_{x, y}^{f}$. Really, when multiplying on $-2 \chi_{22} e_{1 x} e_{1 y}$ the Eq. (20) for $U_{x}^{f}$, and on $\chi_{22}\left(e_{1 y}^{2}-e_{1 x}^{2}\right)$ the Eq. (20) for $U_{y}^{f}$ and adding them at

$\varepsilon_{x x}^{f}=\varepsilon_{y y}^{f}, \sigma_{x x}^{f}=\sigma_{y y}^{f}=\sigma_{\perp}^{f}$

we obtain the equation:

$$
\begin{aligned}
& \frac{\varepsilon_{\perp}^{f}}{4 \pi} \frac{\partial}{\partial t} \Delta \varepsilon_{\perp}+\sigma_{\perp}^{f} \Delta \varepsilon_{\perp}= \\
& =-\beta_{22} \chi_{22}\left(Q^{\prime} A_{1} A_{-1}^{*}-\frac{i \delta}{\chi_{13}} \Delta \varepsilon_{\|}\right) .
\end{aligned}
$$

Eq. (20) for $U_{z}^{f}$ can be multiplied by $\chi_{13}$ and rewritten in the form:

$\frac{\varepsilon_{z}^{f}}{4 \pi} \frac{\partial}{\partial t} \Delta \varepsilon_{\|}+\sigma_{z z}^{f} \Delta \varepsilon_{\|}\left(1-i \delta \frac{\beta_{31}}{\sigma_{z z}^{f}}\right)=$

$=-A_{1} A_{-1}^{*} \chi_{13}\left(\beta_{31} Q^{\prime}+i D^{\prime}\right)$.

The detailed expressions for all the constants in (22)(23) are given below. Let us rewrite the system of Eqs.(4), (22)-(23) in the more convenient form, namely:

$$
\frac{\partial}{\partial z} A_{1}-i p_{0} \Delta_{\perp} A_{1}+(\eta-i \phi) A_{1}=
$$$$
=i\left(W_{\|}+W_{\perp}+\delta W\right) A_{-1} \text {, }
$$

$\frac{\partial}{\partial z} A_{-1}^{*}-i p_{0} \Delta_{\perp} A_{-1}^{*}-\left(\eta+i \phi^{*}\right) A_{-1}^{*}=$

$=i\left(W_{\|}+W_{\perp}+\delta W\right) A_{1}^{*}$,

$\tau_{\perp} \frac{\partial}{\partial t} W_{\perp}+W_{\perp}=-Q_{\perp} A_{1} A_{-1}^{*}+i \delta_{\perp} W_{\|}$,

$\tau_{\|} \frac{\partial}{\partial t} W_{\|}+W_{\|}\left(1-i \delta_{\|}\right)=-\left(Q_{\|}+i D\right) A_{1} A_{-1}^{*}$.

The system (24)-(25) has to be supplemented by initial and boundary conditions. Boundary conditions have the form:

$\left\{\begin{array}{l}A_{1}(z=0, x, y, t)=A_{0}(x, y) \\ A_{-1}(z=\ell, x, y, t)=0 .\end{array}\right.$

Initial conditions must be trivial:

$W_{\|}(t=0)=W_{\perp}(t=0)=0$.

We introduced the following designations in (24)-(25). $p_{0}=1 / 2 k_{0}$,

$W_{\|}=\frac{k_{0}}{2} \Delta \varepsilon_{\|}, \quad W_{\perp}=\frac{k_{0}}{2} \Delta \varepsilon_{\perp}, \quad \delta W=\frac{k_{0}}{2} \delta \varepsilon ;$

$\phi=\frac{k_{0}}{2} \Delta \varepsilon_{P R}$.

The static dielectric permittivity $\widehat{\varepsilon}^{f}$ is uniaxial diagonal tensor: 
A.N. Morozovska, V.V. Obukhovsky: Spatial-temporal peculiarities of periodical PILS ...

$\varepsilon_{x x}^{f}=\varepsilon_{y y}^{f}=\varepsilon_{\perp}^{f}, \quad \varepsilon_{z z}^{f} \neq \varepsilon_{\perp}^{f}, \quad \varepsilon_{z z}^{f} \equiv \varepsilon_{z}^{f}$

Relaxation times could be different

$\tau_{\|}=\varepsilon_{z}^{f} / 4 \pi \sigma_{z z}^{f}, \quad \tau_{\perp}=\varepsilon_{\perp}^{f} / 4 \pi \sigma_{\perp}^{f} ;$

owing to the renormalization of conductivity

$\sigma_{x x}^{f} \approx \sigma_{y y}^{f} \approx \sigma_{\perp}^{f}$

$\sigma_{z z}^{f}=\sigma_{z z}+\frac{k_{0}^{2} \varepsilon_{z}^{f} D_{0}}{\pi} \cdot \frac{\left(\gamma_{k} n_{d s}^{+}+\gamma_{p} I_{s}+\gamma_{T}\right)}{2 \gamma_{k} n_{d s}^{+}+\gamma_{p} I_{s}+\gamma_{T}}$

(notice, that $\sigma_{z z}^{f}>\sigma_{\perp}^{f}$ even in the case $\sigma_{\perp}^{f}=\sigma_{z z}$ );

Coupling constants:

regard scattering intensity much smaller than the pump one. We assume that incline wave $A_{0}$ is the focused gaussian beam in the ideal dielectric medium without absorption. Thus in paraxial approximation $A_{1}$ satisfies the homogeneous equation (24a):

$\frac{\partial A_{1}}{\partial z}-i p_{0} \Delta_{\perp} A_{1}+\Theta(z)(\eta-i \phi) A_{1}=0$

Here $\Theta(z)=\left\{\begin{array}{ll}1, & z>0 \\ 0, & z \leq 0\end{array}\right.$ is theta-function. So $A_{1}=$ $=A_{0}(x, y, z)$ at $z \leq 0$, where:

$A_{1}(x, y, z \leq 0)=\frac{\sqrt{I_{0}}}{1+i n_{0}(z)} \exp \left[-\frac{x^{2}+y^{2}}{2 \rho_{0}^{2}\left(1+i n_{0}(z)\right)}\right]$.

$Q_{\perp}=\frac{k_{0}}{2} \beta_{22} \chi_{22} \frac{\left(n_{d}^{0}-n_{d s}^{+}\right)}{\sigma_{\perp}^{f}} \frac{2 \gamma_{R} n_{d s}^{+}+\gamma_{T}}{2 \gamma_{R} n_{d s}^{+}+\gamma_{p} I_{s}+\gamma_{T}} ;$
$Q_{\|}=\frac{k_{0}}{2} \beta_{31} \chi_{13} \frac{\left(n_{d}^{0}-n_{d s}^{+}\right)}{\sigma_{z z}^{f}} \frac{2 \gamma_{R} n_{d s}^{+}+\gamma_{T}}{2 \gamma_{R} n_{d s}^{+}+\gamma_{p} I_{s}+\gamma_{T}} ;$

$D=\frac{k_{0}}{2} \chi_{13} \frac{\left(n_{d}^{0}-n_{d s}^{+}\right) \gamma_{p}}{\sigma_{z z}^{f}} \frac{2 k_{0} e D_{0}}{2 \gamma_{R} n_{d s}^{+}+\gamma_{p} I_{s}+\gamma_{T}}$

Field interaction constants:

$\delta_{\perp}=\frac{\beta_{22} \chi_{22}}{\sigma_{\perp}^{f} \chi_{13}} \cdot \frac{k_{0} e_{z}^{f}}{2 \pi e} \frac{\gamma_{R} n_{d s}^{+} I_{s}}{2 \gamma_{R} n_{d s}^{+}+\gamma_{p} I_{s}+\gamma_{T}}$,

Here $n_{0}(z)=\frac{z-b_{0}}{k_{0} \rho_{0}^{2}} ; b_{0}$ is the focus "coordinate" regarded from the plain $z=0, a_{0}$ and $F$ is lens aperture and focus distance correspondingly.

Let us approximate the real spatial distribution $\phi(\vec{r})$ by the continuous set of infinitely thin gaussian PR-lenses with refractive index modulation proportional to the pump intensity, because $\Delta \varepsilon_{P R}(\vec{r}) \sim\left|A_{0}(\vec{r})\right|^{2}$. Such optical system is equivalent to the thin lens with thickness $d<<\ell$, diameter $2 \rho_{0}$, effective optical force $\Phi \approx$ $\approx d \sqrt{\Delta \varepsilon_{P R}(0)} /\left(\sqrt{\varepsilon_{0}} \rho_{0}^{2}\right)$ and main plain $z \approx 0$ (see Fig. 2). So, after neglecting the nonlinear terms in (24a), one obtains from (29) at $z \geq d$

$\delta_{\|}=\frac{\beta_{31}}{\sigma_{z z}^{f}} \frac{k_{0} e_{z}^{f}}{2 \pi e} \frac{\gamma_{R} n_{d s}^{+} \cdot I_{s}}{2 \gamma_{R} n_{d s}^{+}+\gamma_{p} I_{s}+\gamma_{T}}$.

Note, if had taken into account $\delta \varepsilon(\vec{r})$ only in Eqs. for laser fields, only the stationary scattering could be obtained because $\delta \varepsilon(\vec{r})$ is time-independent. Dynamical and, in particular, periodical PILS is caused only by the photoinduced fluctuations of the inner field. These fluctuations are proportional to the light intensity and is absent at the initial moment $t=0$. Photoinduced scattering exponentially reinforces in the photoinduced-bleaching regime and therefore it intensity could significantly overcome the stationary scattering one.

\section{Backward periodical PILS in the perturba- tion approach}

\subsection{The pump is focused gaussian beam}

Let us solve system (24) with the boundary conditions (26) for the definite form of $A_{1}$. This means that one could

$S Q O, 6(3), 2003$

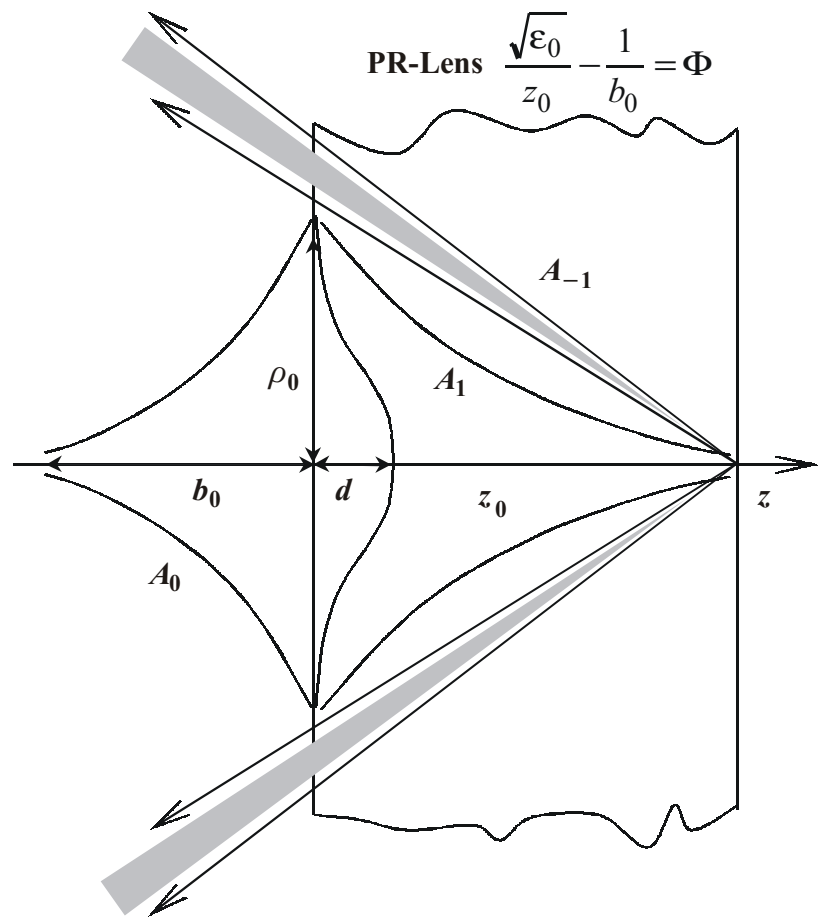

Fig. 2. Gaussian PR-lens in the PRC and autowaves-generation. 
$A_{1}(x, y, z \geq d) \approx$

$\approx \frac{\sqrt{I_{0}}}{1+i n_{f}(z)} \exp \left[-\eta z-\frac{x^{2}+y^{2}}{2 \rho_{0}^{2}\left(1+i n_{f}(z)\right)}\right]$.

Hereinafter function $n_{f}$ depends over $z$ in the following manner $n_{f}(z)=\frac{z-z_{0}}{k_{0} \rho_{0}^{2}} ; z_{0}$ is focus $b_{0}$ "image" regarded from the plain $z=0$.

$z_{0}=\sqrt{\varepsilon_{0}} b_{0} /\left(\Phi b_{0}+1\right)$.

The small term $\sim \Delta \varepsilon_{P R} A_{-1}$ could be neglected in the Eq.(24b) for backward wave $A_{-1}^{*}$. Therefore Eq. (24b) acquires the form:

$\left\{\begin{array}{l}\frac{\partial A_{-1}^{*}}{\partial z}-i p_{0} \Delta_{\perp} A_{-1}^{*}-\eta A_{-1}^{*}=i\left(W_{\|}+W_{\perp}+\delta W\right) A_{1}^{*}(\vec{r}) \\ A_{-1}^{*}(\ell)=0\end{array}\right.$

Hereinafter we use the designation $\tilde{f}(\vec{q}, s, t)$ for Fourier image over $(x, y)$ and Laplace image over $\tilde{z}=\ell-z$ (because $f(\tilde{z}=0) \equiv f(z=\ell)=0)$ of the function $f(x, y, z, t)$, i.e.:

$\tilde{f}(\vec{q}, s, t)=\int_{-\infty}^{\infty} d \vec{q} \exp \left[+i \vec{q} \vec{r}_{\perp}\right] \int_{0}^{\ell-z} d \tilde{z} f(x, y, \tilde{z}, t) \exp [-s \tilde{z}]$.

We would like to underline that the inner field and backward wave sources $\delta \varepsilon$ and $\delta n_{d}$ are random fluctuations. We assume that transverse spatial scale of them is mush smaller than the laser beam diameter $\rho_{0}$. So FourierLaplace image of (33) could be approximated as:

$$
\begin{aligned}
& \tilde{A}_{-1}^{*}(\vec{q}, s, t) \approx-i \sqrt{I_{0}} \exp (-\eta \ell) \times \\
& \times \frac{\tilde{W}_{\|}(\vec{q}, s-\eta, t)+\tilde{W}_{\perp}(\vec{q}, s-\eta, t)+\delta \tilde{W}(\vec{q}, s-\eta)}{\left(1-i\left(\ell-z_{0}\right) / k_{0} \rho_{0}^{2}\right)\left(s+\eta-i q^{2} / 2 k_{0}\right)} .
\end{aligned}
$$

Here $s=\operatorname{Re} s+i \operatorname{Im} s$. Let us find the approximation for convolution $\widetilde{A}_{1} * \widetilde{A}_{-1}^{*}$ used in (25a) and (25b).

Note that for $d<<\ell$ it is necessary $|s|<<q^{2} / 2 k_{0}$. Let us suppose that $|\operatorname{Re} s| \gg>\eta$. In this case it is easy to obtain analogously (35) the following:

$$
\tilde{A}_{1} * \tilde{A}_{-1}^{*} \approx i I_{0} \psi_{z}\left(\tilde{W}_{\|}(\vec{q}, s, t)+\tilde{W}_{\perp}(\vec{q}, s, t)+\delta \tilde{W}_{\perp}(\vec{q}, s)\right)
$$

where

$$
\psi_{z}=-\frac{\exp (-2 \eta \ell)}{1+\left(\ell-z_{0}\right)^{2} / k_{0}^{2} \rho_{0}^{4}} \cdot \frac{1}{s-i q^{2} / 2 k_{0}} .
$$

This is the function of $s, q^{2}$ and $z_{0}$.

Note, that in the case of discrete spatial and temporal spectrum (35) is valid and (34) for the inner field could be represented in the form

$$
W_{\|, \perp}(x, y, z, t)=\sum_{\{\vec{q}, s, \lambda\}} \tilde{W}_{\|, \perp}(\vec{q}, s, t) \exp \left[-i \vec{q} \vec{r}_{\perp}+s(\ell-z)\right],
$$

$\tilde{W}_{\|, \perp}(\vec{q}, s, t)=C_{\|, \perp}(\vec{q}, s, \lambda) \exp [\lambda t]$.

\subsection{Dynamics of the photoinduced field caused by stationary fluctuations of $\delta \varepsilon$}

In this item we perform the bifurcation analyses of system (25) for internal photoinduced field with initial conditions (27). System (25) has been linearized at $I_{s} \rightarrow I_{0}$ and the system for the images $\tilde{W}_{\|, \perp}$ has been obtained allowing for (36)-(38), namely:

$$
\left\{\begin{array}{l}
\tau_{\|} \frac{\partial \tilde{W}_{\|}}{\partial t}+\left(1-i \delta_{\|}\right) \tilde{W}_{\|}= \\
=-i\left(Q_{\|}+i D\right) I_{0} \psi_{z}\left(\tilde{W}_{\|}+\tilde{W}_{\perp}\right)-i I_{0} \tilde{R}_{\|}, \\
\tau_{\perp} \frac{\partial \tilde{W}_{\perp}}{\partial t}+\tilde{W}_{\perp}= \\
=-i Q_{\perp}\left(\tilde{W}_{\|}+\tilde{W}_{\perp}\right) I_{0} \psi_{z}+i \delta_{\perp} \tilde{W}_{\|}-i I_{0} \tilde{R}_{\perp}, \\
\tilde{W}_{\|}(0)=0, \quad \tilde{W}_{\perp}(0)=0 .
\end{array}\right.
$$

Here we used the designations

$\tilde{R}_{\|}(\vec{q}, s)=\left(Q_{\|}+i D\right) \psi_{z} \delta \tilde{W}(\vec{q}, s)$,

$\tilde{R}_{\perp}(\vec{q}, s)=Q_{\perp} \psi_{z} \delta \tilde{W}(\vec{q}, s)$.

It is clear from (40) that the sources of photoinduced field are proportional to $\delta \varepsilon$ static fluctuations. Thus the sources (40) are stationary and weakly depend over intensity $I_{0}$ at $\gamma_{p} I_{0}>>\gamma_{T}$.

The solution of system (40) has the form:

$$
\begin{aligned}
& \tilde{W}_{\|}=I_{0}\left[R_{1}+\frac{R_{1} \alpha_{2}-R_{2}}{\alpha_{1}-\alpha_{2}} \exp \left[\lambda_{1} t\right]+\right. \\
& \left.+\frac{R_{2}-\alpha_{1} R_{1}}{\alpha_{1}-\alpha_{2}} \exp \left[\lambda_{2} t\right]\right], \\
& \tilde{W}_{\perp}=I_{0}\left[R_{2}+\alpha_{1} \frac{R_{1} \alpha_{2}-R_{2}}{\alpha_{1}-\alpha_{2}} \exp \left[\lambda_{1} t\right]+\right. \\
& \left.+\alpha_{2} \frac{R_{2}-\alpha_{1} R_{1}}{\alpha_{1}-\alpha_{2}} \exp \left[\lambda_{2} t\right]\right] .
\end{aligned}
$$

In (41) we used the designations 


\section{A.N. Morozovska, V.V. Obukhovsky: Spatial-temporal peculiarities of periodical PILS ...}

$\alpha_{m}=i \frac{\delta_{\perp}-Q_{\perp} I_{0} \psi_{z}}{1+i Q_{\perp} I_{0} \psi_{z}+\tau_{\perp} \lambda_{m}}, \quad m=1,2$

$R_{1}=\frac{i}{\Delta_{P}} \cdot\left[\tilde{R}_{\perp}\left(i Q_{\|}-D\right) I_{0} \psi_{z}-\tilde{R}_{\|}\left(1+i Q_{\perp} I_{0} \psi_{z}\right)\right]$

$R_{2}=\frac{i}{\Delta_{P}}\left[i \tilde{R}_{\|}\left(Q_{\perp} I_{0} \psi_{z}-\delta_{\perp}\right)-\right.$

$\left.-\tilde{R}_{\perp}\left(1-i \delta_{\|}+\left(i Q_{\|}-D\right) I_{0} \psi_{z}\right)\right]$,

where

$$
\begin{aligned}
& \Delta_{P}=\left(1-i \delta_{\|}+\left(i Q_{\|}-D\right) I_{0} \psi_{z}\right)\left(1+i Q_{\perp} I_{0} \psi_{z}\right)- \\
& -i\left(Q_{\perp} I_{0} \psi_{z}-\delta_{\perp}\right)\left(i Q_{\|}-D\right) .
\end{aligned}
$$

Eigen values $\lambda_{m}$ are the roots of the quadratic equation, which can be obtained from the condition of zero determinant of system (25):

$\operatorname{det} \hat{H}=0$,

$\hat{H}=\left(\begin{array}{ll}\tau_{\|} \lambda+1-i \delta_{\|}+\left(i Q_{\|}-D\right) I_{0} \psi_{z} & \left(i Q_{\|}-D\right) I_{0} \psi_{z} \\ i Q_{\perp} I_{0} \psi_{z}-i \delta_{\perp} & \tau_{\perp} \lambda+1+i Q_{\perp} I_{0} \psi_{z}\end{array}\right)$.

\subsection{The absolute instability threshold}

The quadratic equation $\operatorname{det} \hat{H}=0$ can be rewritten as

$\lambda^{2}+a \lambda+b=0$, from $\lambda_{1,2}=-\frac{a}{2} \pm \sqrt{\frac{a^{2}}{4}-b}$.

Here

$a=\frac{1-i \delta_{\|}+I_{0} \psi_{z}\left(i Q_{\|}-d\right)}{\tau_{\|}}+\frac{1+I Q_{\perp} I_{0} \psi_{z}}{\tau_{\perp}}$,

$b=\frac{1}{\tau_{\|} \tau_{\perp}} \Delta_{P}$.

The condition $\operatorname{Re}\left(\lambda_{m}\right)=0$ is the instability threshold. The absolute instability threshold is the following:

$\operatorname{Re}\left(\lambda_{1}\right)=\operatorname{Re}\left(\lambda_{2}\right)=0$.

The threshold (46) reaches under conditions:

$\operatorname{Re}(a)=0, \quad \operatorname{Im}(b)=0, \quad \operatorname{Re}(b)+\frac{\operatorname{Im}^{2}(a)}{4}>0$.

In such case the self-oscillations with frequencies $\Omega_{1,2}$ do realize:

$\lambda_{1,2}=i \Omega_{1,2}$,

$\Omega_{1,2}=-\frac{\operatorname{Im}(a)}{2} \pm \sqrt{\operatorname{Re}(b)+\frac{\operatorname{Im}^{2}(a)}{4}}$.
The complex value $\psi_{z}=\operatorname{Re} \psi_{z}+i \operatorname{Im} \psi_{z}$, i.e. $s, q^{2}$, must be determined from Eqs. (47). This solution can be represented as:

$\operatorname{Re} \psi_{z}=f_{1} / I_{0}, \quad \operatorname{Im} \psi_{z}=f_{2} / I_{0}$,

where

$$
\begin{aligned}
& f_{1}=\left[\left(\delta_{\|} Q_{\perp}-D-\delta_{\perp} Q_{\|}\right)\left(1+\frac{\tau_{\|}}{\tau_{\perp}}\right)-\right. \\
& \left.-\left(Q_{\|}+\frac{\tau_{\|}}{\tau_{\perp}} Q_{\perp}\right) \delta_{\|}\right] \frac{1}{\Delta_{\psi}},
\end{aligned}
$$

$f_{2}=\left[\delta_{\|} D-\left(1+\frac{\tau_{\|}}{\tau_{\perp}}\right)\left(Q_{\perp}+Q_{\|}-\delta_{\perp} D\right)\right] \cdot \frac{1}{\Delta_{\psi}}$

$\Delta_{\psi}=D\left(\delta_{\|} Q_{\perp}-D-\delta_{\perp} Q_{\|}\right)-$

$-\left(Q_{\|}+\frac{\tau_{\|}}{\tau_{\perp}} Q_{\perp}\right)\left(Q_{\|}+Q_{\perp}-\delta_{\perp} D\right)$.

The expressions for the autowaves frequencies $\Omega_{1,2}$, absolute value of the transverse wave vector $q_{0}^{2}$ and spatial gain factor $s_{0}$ have been obtained from (38), (48)(50).

Let us present and analyze the expressions for $\Omega_{1,2}$, $q_{0}^{2}, s_{0}$ derived under the approximation

$\tau_{\|}<<\tau_{\perp}, \quad \delta_{\|, \perp} \rightarrow 0, \quad\left(|D|+\left|Q_{\perp}\right|\right)<<\left|Q_{\|}\right|$.

Really, when used (38), (48)-(53), one can obtain relatively simple expressions for $\Omega_{1,2}, q_{0}^{2}, s_{0}$, namely:

$\Omega_{1} \equiv \Omega, \quad \Omega \approx \frac{1}{\tau_{\|}} \frac{\left|D Q_{\perp}\right|}{Q_{\|}^{2}}, \quad \Omega_{2} \approx 0$,

$q_{0}^{2} \approx-\frac{2 k_{0} I_{0} Q_{\|} \exp (-2 \eta \ell)}{1+\left(\ell-z_{0}\right)^{2} / k_{0}^{2} \rho_{0}^{4}}$,

$s_{0}=-\frac{I_{0} D \exp (-2 \eta \ell)}{1+\left(\ell-z_{0}\right)^{2} / k_{0}^{2} \rho_{0}^{4}}$.

The following important for the comparison with the experiment conclusions could be made from (54)-(56).

1 . Let us study the dependence $\Omega_{1} \equiv \Omega\left(I_{0}\right)$ in accordance with (54). At rather high intensities $\gamma_{p} I_{0} \gg \gamma_{T}$ : $1 / \tau_{\|} \sim \sigma_{\|} \sim n_{e}^{s} \sim I_{0}$, and so $\Omega \sim I_{0}$. Under further intensity increasing $n_{e}^{s} \rightarrow n_{d}^{0}-n_{a}$ and frequency (54) saturates. The similar speculations for the transverse wave vector lead to the dependence $q_{0} \sim \sqrt{I_{0}}$, but at very high intensities $q_{0}$ tends to the constant value.

2. As it follows from (55), the condition $Q_{\|}<0$ is necessary for periodic PILS observation. This restricts 
the type of PR-materials in which optical autowaves could exist.

3. Note, that $W_{\|, \perp}(x, y, z, t) \sim \tilde{W}_{\|, \perp}(t) \exp \left[i \vec{q} \vec{r}_{\perp}\right] \times$ $\times \exp \left[+s_{0}(\ell-z)\right]$, and so the condition $\exp \left[s_{0} \ell\right]>>1$ as well as $D<0$, is urgent for the weak backward wave amplification (see (56)). The condition $D<0$ corresponds to the definite pump beam propagation direction along polar axes $z$, because $D \sim D_{0} \chi_{13}$ changes its sign at crystal rotation over $180^{\circ}(z \rightarrow-z)$.

4. We'd like to underline that expression (55) determines only the absolute value $q_{0}$ of the vector $q$. Thus the periodic process could origin with all transverse vectors, that satisfy the equation $\vec{q}^{2}=q_{0}^{2}$, i.e. there is no reason to suppose, that any of possible wave vector directions is preferable.

\subsection{Optical autowaves}

Notice that in photoinduced-bleaching regime in rather thick samples with small absorption, i.e. at

$\exp \left(\frac{-I_{0} D \ell \exp (-2 \eta \ell)}{1+\left(\ell-z_{0}\right)^{2} / k_{0}^{2} \rho_{0}^{4}}\right)>>1$,

stationary scattering on the fluctuations proportional to $\delta \tilde{W}$, can be neglected at $z=0$. In the case the spatial gain factor for scattering at the photoinduced fluctuations of internal field is proportional to the $\tilde{W}_{\|, \perp} \exp \left[s_{0} \ell\right] \sim \delta \tilde{W} \exp \left[s_{0} \ell\right]$, which is much more than unity.

If $\rho_{0}^{2} q_{0}^{2}>>1$, the backward wave does not interact with the PR lens with diameter $\rho_{0}$ (see Fig. 2). Using Eqs (33), (35), (38), (57), one can obtain the solution for the backward wave field at the front plane of the sample $(z=0)$ in the following form:

$$
\begin{aligned}
& A_{-1}^{*}(x, y, 0, t) \approx \frac{-i \sqrt{I_{0}}}{1-i\left(\ell-z_{0}\right) / k_{0} \rho_{0}^{2}} \frac{\exp \left[s_{0} \ell\right]}{s_{0}-i q_{0}^{2} / 2 k_{0}} \times \\
& \times \sum_{\vec{q}^{2}=q_{0}^{2}} \exp \left(\vec{q} \cdot \vec{r}_{\perp}\right)\left(\tilde{W}_{\|}\left(\vec{q}, s_{0}, t\right)+\tilde{W}_{\perp}\left(\vec{q}, s_{0}, t\right)\right) .
\end{aligned}
$$

Taking into account Eqs. (54), (48) the solution of (41) can be found as follows:

$$
\begin{aligned}
& \tilde{W}_{\|}\left(\vec{q}, s_{0}, t\right)=2 I_{0} \frac{R_{1} \alpha_{2}-R_{2}}{\alpha_{1}-\alpha_{2}} \exp \left[i \frac{\Omega}{2} t\right] \cdot \sin \left(\frac{\Omega}{2} t\right), \\
& \tilde{W}_{\perp}\left(\vec{q}, s_{0}, t\right)=\alpha_{1} \tilde{W}_{\|} .
\end{aligned}
$$

Here $\vec{q}^{2}=q_{0}^{2}$. Allowing for the analyses of the item 2.3, we postulate that

$$
\begin{aligned}
& \left\langle\tilde{W}\left(\vec{q}, s_{0}, t\right)\right\rangle=0, \\
& \left\langle\tilde{W}\left(\vec{q}, s_{0}, t\right) \tilde{W}^{*}\left(\vec{q}^{\prime}, s_{0}, t\right)\right\rangle \sim \delta\left(\vec{q}-\vec{q}^{\prime}\right)\left\langle\left|\tilde{W}\left(q_{0}, s_{0}, t\right)\right|^{2}\right\rangle .
\end{aligned}
$$

Angle brackets denote the averaging over random sources $R_{1,2}$ (see. (42b)), $\alpha_{1,2}$ is given by expression (42a). So, the summarization over $\vec{q}$ directions is substituted by the averaging with uniform distribution function.

The backward wave amplitude $E_{-1}$ on the screen with coordinates $\left\{x_{S}, y_{S}, z_{S}=-L\right\}, L>>1$, has been calculated in paraxial approximation by means of Kirghoff's integral [16]. The gaussian profile of the pump wave has been taken into account. The calculation is represented in Appendix1. In the case $\rho_{0}^{2}<<L / k_{0}$ the backward autowaves' PILS indicatrix at angle $\theta$ is equal to:

$$
j_{-1}(\theta) \approx R_{0}^{2}(1-\cos (\Omega t)) \frac{\exp \left\lfloor-\Delta^{2}\left(\theta-\theta_{0}\right)^{2} / 2\right\rfloor}{1+\pi \Delta^{2} \theta_{0} \cdot \theta} \text {. }
$$

In Eq.(60) the following designations are introduced:

$$
\begin{aligned}
& R_{0}^{2} \approx\left\langle\left.\left(1+\alpha_{1}\right) \frac{R_{1} \alpha_{2}-R_{2}}{\alpha_{1}-\alpha_{2}}\right|^{2}\right\rangle\left(\frac{k_{0} \rho_{0}^{2}}{2 L}\right)^{2} \times \\
& \times \frac{1+\left(\ell-z_{0}\right)^{2} / k_{0}^{2} \rho_{0}^{4}}{D^{2}+Q_{\|}^{2}} \times \\
& \times \exp \left[-\frac{2 I_{0} D \ell \exp (-2 \eta \ell)}{1+\left(\ell-z_{0}\right)^{2} / k_{0}^{2} \rho_{0}^{4}}\right], \\
& j_{-1}(\theta, t)=I_{-1}(\theta, t) / I_{0}, \quad \theta_{0}=q_{0} / k_{0}, \\
& \Delta=k_{0} \rho=\frac{F}{a_{0}}, \quad \rho^{2}=\rho_{0}^{2}\left(1+n_{f}^{2}(0)\right),
\end{aligned}
$$

It is follows from the analysis of (60) and numerical calculation with the help of Eq.(A.9) that for the thick enough sample backward scattering has the conic space structure with angle $\theta_{0}$ and periodically varies in time with the frequency $\Omega$. On a screen the profiles of the averaged scattering indicatrix (60) have the view of periodically appearing rings that are well resolved in the backward experiment geometry when the central maximum is absent.

\section{The characteristics of the scattered irradia- tion. Comparison with the experiment on peri- odic PILS in $\mathrm{LiNbO}_{3}: \mathrm{Fe}$}

Notice, that theoretically calculated diffraction pattern for backward scattering, at least for the "thick" crystal, possesses significantly higher visualization, than the same one for the forward PILS, which can be calculated in only the next order of perturbation theory. So we restrict ourselves by analysis of those basic characteristics of inverse scattering, which have been compared with the available experimental data on autowave PILS in $\mathrm{LiNbO}_{3}$ crystals, doped with $0.02 \div 0.07$ wt. $\%$ Fe intensively studied in our lab. Hereinafter we assume that it is possible to 


\section{A.N. Morozovska, V.V. Obukhovsky: Spatial-temporal peculiarities of periodical PILS ...}

neglect the dark conductivity due to sufficient laser intensity $\gamma_{p} I_{0} \gg \gamma_{T}$, i.e. to substitute $\gamma_{T}=0$. Also the experiments were carried out at the "optimal focusing conditions", i.e. at $\left(\ell-z_{0}\right)^{2} / k_{0}^{2} \rho_{0}^{4}<<1$.

We would like to remind that backward PILS has the conic spatial structure with the cone opening oscillating around $\theta_{0}$ (see (60)). It is appeared convenient to characterise these temporal oscillations by the minimum angle $\theta_{\min }$ (i.e. the angle of cone generation), the angle of the best visualisation $\theta_{0}$ (i.e. the angle of cone maximum intensity), and the maximum angle $\theta_{\max }$ (i.e. the angle of cone disappearance). The experimentally measured values of $\theta_{\max }$ and $\theta_{\min }$ can be additionally limited by the resolution of the register hardware. We determined these angles numerically from (60). It is turned out that for a wide region of parameters with high accuracy one can apply clear and simple relations:

$$
\theta_{0} \approx\left(\theta_{\max }+\theta_{\min }\right) / 2, \quad \theta_{\min } \approx H \theta_{0}, \quad \theta_{\max } \approx(2-H) \theta_{0} .
$$

Where $0<H<1$ is positive constant. Thus the cone parameters are determined by the angle $\theta_{0}$, which is proportional to $q_{0} \sim \sqrt{Q_{\perp}} \sim \sqrt{\left(n_{d}^{0}-n_{d s}^{+}\right)}$in accordance with (55) and (28d). As it follows from (15) it increases with the increasing of the photoactive impurity concentration $n_{d}^{0}$ as $\theta_{0} \sim \sqrt{n_{d}^{0}-n_{a}}$. We denote that $\left(n_{d}^{0}-n_{a}\right)=n_{0}\left(N_{d}-N_{a}\right)$, where the concentration of donors $N_{d}$ and acceptors $N_{a}$ is measured in \% of matrix concentration $n_{0}$. Finally one obtains:

$\theta_{0} \approx \theta_{f} \sqrt{N_{d}-N_{a}}$,

$\theta_{f} \approx \sqrt{\frac{I_{0}}{1+\left(\ell-z_{0}\right)^{2} / k_{0}^{2} \rho_{0}^{4}} \frac{n_{0} \beta_{31} \chi_{13}}{\sigma_{z z}^{f}} \frac{\gamma_{R} n_{a}}{\gamma_{R} n_{a}+\gamma_{P} I_{0}+\gamma_{T}}}$.

Calculated in accordance with (63) dependences of the angles $\theta_{\min }, \theta_{0}$ and $\theta_{\max }$ over impurity concentration $N_{d}$ have been compared with the experimentally obtained ones in Fig. 3.

Let us discuss the temporal spectrum of scattering waves. The theoretical (see (54)) and experimental dependences of autowaves' frequency $\Omega$ over pump wave intensity $I_{0}$ are presented in Fig. 4. To all appearances we possess the experimental data for the linear part of the aforementioned dependence, i.e. for high-quality samples and rather high $I_{0}$, where

$$
\Omega\left(I_{0}\right)=\gamma_{f} I_{0} \text {. }
$$

The investigation of the process at smaller intensities proves the existence of intensity threshold: the autowaves' PILS loses its periodical structure and eventually splits into random flickering spots at intensities smaller that the critical intensity $I_{\mathrm{C}}$. Critical intensity $I_{\mathrm{C}}$ could be es-

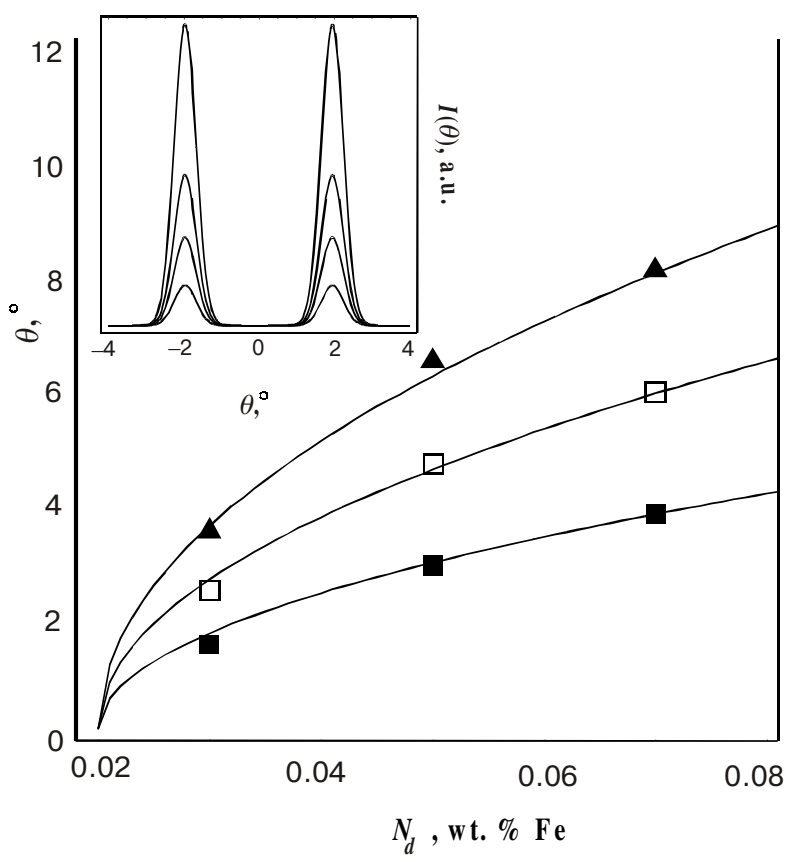

Fig. 3. The dependences of scattering cone angles $\theta_{\min }$ (filled squares), $\theta_{0}$ (empty squares), $\theta_{\max }$ (empty triangles) over Fe concentration in $\mathrm{LiNbO}_{3}$. The best agreement with the experiment was obtained at $\theta_{f}=26.5^{\circ}, N_{a}=0.02 \%, H=0.64$. Inset: the angular structure of the autowaves' intensity on the screen

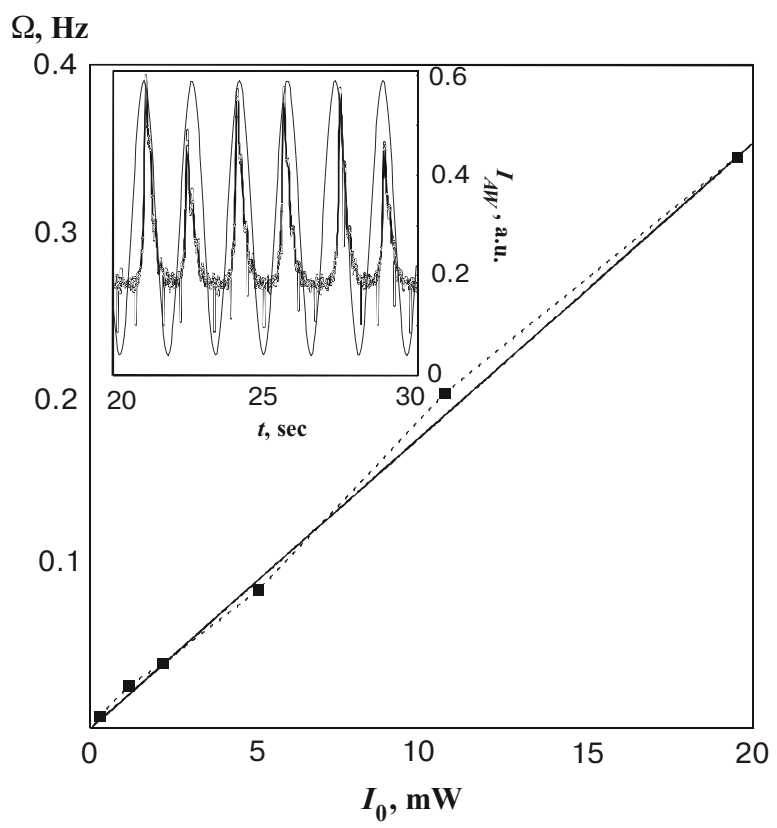

Fig. 4. Theoretical (solid curve, calculated by Eq.(65) at $\gamma_{f}=$ $=0.018 \mathrm{~mJ}^{-1}$ ) and experimental (filled squares, $N_{d}=0.03 \%$ ) dependencies of autowaves frequency $\Omega$ over $I_{0}$. Inset: the experimental (bold curve) and theoretical (fine curve) dependences of the averaged over the ring autowave intensity over time. 
timated from the conditions $s_{0} \ell>1$ and $s_{0}>2 \eta$ (see (56), (61)). At $\left(\ell-z_{0}\right)^{2} / k_{0}^{2} \rho_{0}^{4}<<1$ the estimation for $I_{\mathrm{C}}$ acquires the form:

$$
I_{C} \cong-\frac{1+2 \eta \ell}{2 D \ell} \exp (2 \eta \ell)
$$

i.e. critical intensity is inversely proportional to the diffusion coefficient.

Within the framework of our theory the autowaves' frequency does not depend on the crystal thickness. Possibly this dependence is present below the intensity threshold. The temporal dependence of the autowaves intensity averaged over ring can be easily calculated from (60), namely it obeys the law $(1-\cos (\Omega t))$. In comparing the result with the experimentally obtained autowaves evolution (see inset in Fig. 4), to our mind, the qualitatively compliance in general dynamics of the process is evident, the differences of the experimental and theoretical pro-
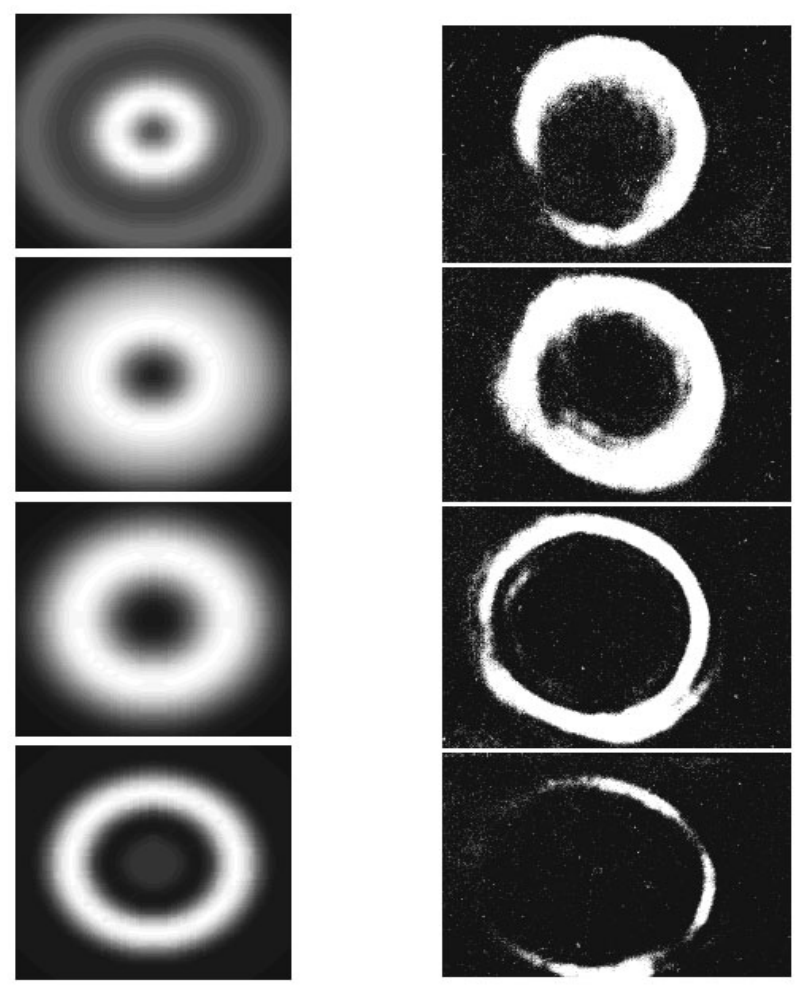

Fig. 5. Dynamics of autowaves on the screen S: appearance, spreading out, disappearance. Theoretical calculations (left) and experimental observation (right). files on the period is related with the linearity of the theory. In fact we omitted all non-linear terms in the initial system, i.e. neglected the possibility of the crystal to generate sub- and super- harmonics.

The most simple for the analysis and the best corresponded with the experiment is the dynamics of the rings of backward autowave PILS on a screen (see Fig. 5). In this case rather bright ring appears on a screen, then extends with decreasing of its thickness and gradually disappears. After this the crystal generates the following ring.

Let us calculate such important characteristic as the spatial correlation coefficient of the backward scattering, hereinafter designated as $K_{a b}$. Namely let us derive the correlation function of the two points " $a$ " and " $b$ " on the screen (characterised by spherical angles $\vec{\theta}_{a}=\left(\theta_{a}, \varphi_{a}\right)$ and $\left.\vec{\theta}_{b}=\left(\theta_{b}, \varphi_{b}\right)\right)$ with different (in general) time-averaged intensities $I_{-1}\left(\vec{\theta}_{a}\right)=\overline{I_{-1}\left(\vec{\theta}_{a}, t\right)}$ and $I_{-1}\left(\vec{\theta}_{b}\right)=\overline{I_{-1}\left(\vec{\theta}_{b}, t\right)}$.

By definition

$$
K_{a b}=\frac{2 I_{-1}\left(\vec{\theta}_{a}\right) I_{-1}\left(\vec{\theta}_{b}\right)}{\left(I_{-1}\left(\vec{\theta}_{a}\right)\right)^{2}+\left(I_{-1}\left(\vec{\theta}_{b}\right)\right)^{2}} .
$$

In fact we calculate $K_{a b}$ near the generation threshold, where inequality (57) is not strong, and it is necessary to take into account the stationary scattering with intensity $I_{0} R_{\mathcal{E}}^{2}(\theta, \varphi)$. When

$\theta_{a} \approx \theta_{b} \approx \theta_{0}, \quad \varphi_{a} \neq \varphi_{b}, \quad I_{0} \sim I_{C}$.

In accordance with (60) and (68a):

$I_{-1}\left(\vec{\theta}_{a}\right)=I_{0} R_{0}^{2}+I_{0} R_{\mathcal{E}}^{2}\left(\theta_{0}, \varphi_{a}\right)$,

$I_{-1}\left(\vec{\theta}_{b}\right)=I_{0} R_{0}^{2}+I_{0} R_{\mathcal{E}}^{2}\left(\theta_{0}, \varphi_{b}\right)$,

$R_{0}^{2}=\widetilde{R}_{0}^{2} \exp \left[-2 I_{0} D\left(I_{0}\right) \ell\right]$,

$D\left(I_{0}\right)=\frac{\tilde{D}}{\left(1+I_{0} / I_{d}\right)^{2}}$,

$I_{d} \approx \frac{\gamma_{P}}{2 \gamma_{R} n_{a}}$

The approximation for the dependence of generalized diffusion coefficient $D\left(I_{0}\right)$ over intensity was obtained from (15) and (28f). Thus $K_{a b}$ acquires the form:

$$
\begin{gathered}
K_{a b}=1-\frac{\left(1-R_{a b}\right)^{2}}{\left(1+R_{0 b} \exp \left[-2 I_{0} \tilde{D} \ell /\left(1+I_{0} / I_{d}\right)^{2}\right)^{2}+\left(R_{a b}+R_{0 b} \exp \left[-2 I_{0} \tilde{D} \ell /\left(1+I_{0} / I_{d}\right)^{2}\right)^{2}\right.\right.}, \\
R_{a b}=\frac{R_{\mathcal{E}}^{2}\left(\theta_{0}, \varphi_{a}\right)}{R_{\mathcal{E}}^{2}\left(\theta_{0}, \varphi_{b}\right)}, \quad R_{0 b}=\frac{R_{0}^{2}}{R_{\mathcal{E}}^{2}\left(\theta_{0}, \varphi_{b}\right)} .
\end{gathered}
$$




\section{A.N. Morozovska, V.V. Obukhovsky: Spatial-temporal peculiarities of periodical PILS ...}

In the case typical for the incoherent isotropic stationary scattering, $R_{a b}<<1$ and $R_{0 b}<<1$, if only detectors are far enough from each other. So (69) can be simplified as

$$
\begin{aligned}
& K_{a b}(\varphi)=\frac{K_{0}(\varphi) \exp \left[G I_{0} /\left(1+I_{0} / I_{d}\right)^{2}\right]}{1+K_{0}(\varphi) \exp \left[G I_{0} /\left(1+I_{0} / I_{d}\right)^{2}\right]} \\
& G=-2 \tilde{D} \ell, \quad K_{0}(\varphi)=2 R_{0}^{2} / R_{\mathcal{E}}^{2}\left(\theta_{0}, \varphi_{b}\right) .
\end{aligned}
$$

The value $K_{0}<<1$ is independent over the angle $\varphi$ between detectors for isotropic stationary scattering.

The value $G>0$ in photoinduced-bleaching regime, and $K_{a b}$ saturates under intensity $I_{0}$ increasing as it should be expected. The dependence of the spatial correlation coefficient $K_{a b}$ over intensity $I_{0}$ and comparison with obtained experimental data are represented in Fig. 6. The amplitude $K_{0}$, saturation constant $I_{d}$ and gain factor $G$ are fitting parameters. Under comparing with the experiment the dependence $K_{a b}$ over $I_{0}$ we used (70), and therefore considered the register detectors placed on the ring in accordance with (68) and assumed that they were the point ones (i.e. we approximate the real apparatus function by Dirac delta-function). The latter approximation is pertinent only if $\varphi_{d}<<\left|\varphi_{b}-\varphi_{a}\right|\left(\varphi_{d}\right.$ is the angular detector size).

We would like to stress that autowaves' PILS was observed only in the case of focused (but not the plain)

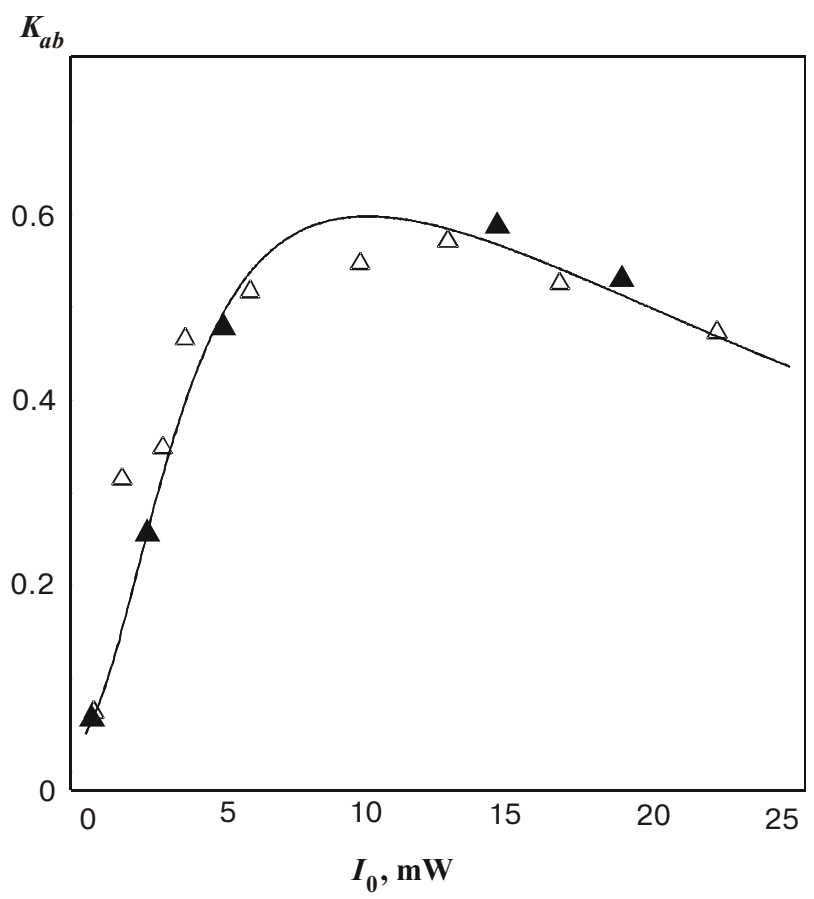

Fig. 6. The dependences of the measured and calculated at $K_{0}=0.04$ and $G=1.43 \mathrm{~mW}^{-1}, I_{d}=10 \mathrm{~mW}$ spatial correlation coefficient $K_{a b}$ over intensity $I_{0}$ at the different angle between the detectors: $\varphi=90^{\circ}$ (empty triangles), $\varphi=180^{\circ}$ (filled triangles).
Gaussian pump beam with the beam focus located directly in front of the PRC. Really, gain factor (56) is maximal under the condition $z_{0}=\ell$, which in accordance with (32) corresponds to the following focus coordinate $b_{0}=-\ell /\left(\Phi \ell-\sqrt{\varepsilon_{0}}\right)$ relatively of the front plane. The condition $b_{0}<0$ satisfies if $\Phi \ell>\sqrt{\varepsilon_{0}}$. Assuming that effective optical force has the view $\Phi \sim$ $\sqrt{\Delta \varepsilon_{P R}} \ell /\left(\sqrt{\varepsilon_{0}} \rho_{0}^{2}\right)$, we have done some estimations. At $\sqrt{\varepsilon_{0}} \sim 1, \rho_{0} \approx 5 \cdot 10^{-3} \mathrm{~cm}, \sqrt{\Delta \varepsilon_{P R}} \approx 5 \cdot 10^{-4}, \ell \approx 0.5 \mathrm{~cm}$ one obtains $\Phi \approx 10 \mathrm{~cm}^{-1}, b_{0} \sim-0.1 \mathrm{~cm}$. In principle, the effective value $\Phi$ can be regarded as fitting parameter.

Note, that autowaves' PILS was found only in the photoinduced-bleaching regime. All the dynamic effects disappear in the reflecting regime, i.e. under turning the sample over $180^{\circ}$ in XY-plane. This fact confirms the validity of the corresponding speculations from the item 2.3 (see (56)).

The refractive index of the outer medium doesn't influence on the existence of autowaves: the crystal generates them both in the air and in the immersion or even in the water. This confirms the assumption (see 1.1-1.2) about the fact that the seeding for the backward wave is "bulk" sources $\delta \varepsilon(\vec{r})$.

The hypothesis (see 1.1) about the coincidence of the pump (direct) wave polarization and backward scattered one was confirmed experimentally with a high accuracy up to angular seconds.

The periodic PILS has been registered only in the high-quality $\mathrm{LiNbO}_{3}$ single crystals doped with Fe. Similar effects have not been found in any other samples. In accordance with the expression (54), we explain these results by the presence of the non-zero component $\beta_{\perp}$ only in the ilmenite $\mathrm{LiNbO}_{3}$ and its absence in all other investigated samples.

Thus, though the real temporal spectrum of PILS has been approximated by delta-function in our linearized theory, the main spatial and temporal characteristics of the process obtained in this way are in a full agreement with the available experimental data on optical autowaves observation.

\section{Conclusions}

In this paper we have studied consecutively the optical autowaves generation in the uniaxial ferroelectric PRC under stationary laser irradiation. The characteristic features of the backward PILS have been predicted. The obtained results can be briefly formulated as following statements.

For the first time it has been shown that, due to influence of transverse photovoltaic current and diffusion, among all possible scenarios of transverse instabilities the boundary circle will be realized.

Due to this phenomenon the periodical or quasiperiodical PILS appear in the real system, depending on the structure of growth non-homogeneities and the character of photoinduced fluctuations caused by them. 
- The optical autowaves generation is possible in such uniaxial ferroelectric PRC as ilmenites under stationary laser irradiation.

All the main theoretical results are in a good agreement with the available experimental data on autowaves' PILS in $\mathrm{LiNbO}_{3}$ crystals, doped with $0.02 \div 0.07$ wt. $\%$ $\mathrm{Fe}[1]$.

\section{Acknowledgements}

The authors are greatly indebted to Dr. V.V. Lemeshko for experimental data used in the paper. The work is supported by grant INTAS-01-0173.

\section{References}

1. V.V. Lemeshko, V.V. Obukhovskii, Autowaves of photoinduced light scattering // Pis'ma. Zh. Tekh. Fiz., 11(11), pp. 1389-1393 (1985).

2. A.S. Furman, Spontaneous increasing of recharging waves in crystals without inversion center under stationary illumination // Sol. St. Phys., 29(4), pp. 1076-1085 (1987).

3. B.I. Sturman, E. Sharmonina, M. Mann, K.H. Ringhofer, Space-charge waves in photorefractive ferroelectrics // J Opt. Soc. Am., 12(9), pp. 1642-1650 (1995).

4. A.S. Furman, Dynamics of recharging waves // JETP 94 pp.295-300 (1988)

5. D. Engin, S. Orlov, M. Segev, G.C. Valley, A. Yariv, Orderdisorder phase transition and critical slowing down in photorefractive self-oscillators // Phys.Rev. Lett., 74(10), pp. 1743-1746 (1995).

6. O. Sandfuchs, J. Leonardy, F. Kaiser and M. Belic, Transverse instabilities in photorefractive counter propagating twowave mixing // Phys. Rev., A53(6), pp.4519-4524 (1996.)

7. B.I. Sturman, E. Sharmonina, M. Mann, K.H. Ringhofer, Space-charge waves in photorefractive ferroelectrics // $J$. Opt. Soc. Am., 12(9), pp. 1672-1650 (1995).

8. N.N. Rozanov, Hysteresis phenomena in distributed optical systems // JETP., 80(1), pp.96-108 (1981).

9. A.N.Morozovska, V.V. Obukhovskii, Autowave-type instability in photorefractive crystals // Proceedings of SPIE, 3904, pp. 209-214 (1999).

10. A.N.Morozovska, V.V. Obukhovskii, V.V. Lemeshko, Dynamics of Photoinduced Instability in Ferroelectric Photorefractive Crystals // Semiconductor Physics, Quantum Electronics \& Optoelectronics, 4(4), pp.358-374 (2001).

11. A.N.Morozovska, V.V. Obukhovskii, V.V. Lemeshko, Dynamics of Photoinduced Instability in Ferroelectric Photorefractive Crystals Caused by Recharging Trap Waves // Semiconductor Physics, Quantum Electronics \& Optoelectronics, 5(3), pp. 268-283 (2002).

12. N.V. Kukhtarev, V.B. Markov, S.G. Odulov, M.M. Soskin, V.L. Vinetskii, Holographic Storage in Electrooptic crystals // Ferroelectrics, 22, pp. 949-960 (1979).

13. V.M. Fridkin, Photo-Ferroelectrics, Nauka, Moscow (1979) (in Russian).

14. M.E.Lines and A.M.Glass, Principles and Applications of Ferroelectrics and Related Phenomena, Oxford University Press (1978)

15. U.S. Kuzminov, Litium niobate and tantalate are the materials for nonlinear optics, Nauka, Moscow (1975) (in Russian).

16. T.S. Yu, Introduction to diffraction, information processing and holography, MIT Press Cambridge, Massachusetts and London (1973).

\section{Appendix A. Far field PILS indicatrix}

The field of the backward wave $E_{-1}$ on the screen with the coordinates $\left\{x_{S}, y_{S}, z_{S}=-L\right\}, L>>1$, in paraxial approximation should be calculated with the help of Kirghoff's formula:

$E_{-1}(\vec{\theta}, z=L, t)=$

$=\frac{i k_{0}}{4 \pi} \int_{-\infty}^{\infty} d x d y \frac{\exp \left[i k_{0} r\right]}{r} A_{-1}(x, y, z=0, t)$.

Here the following designation are introduced:

$\left\{\begin{array}{l}r=\sqrt{L^{2}+\left(x_{S}-x\right)^{2}+\left(y_{S}-y\right)^{2}} \approx \\ \approx L+\left(x_{S}-x\right)^{2} / 2 L+\left(y_{S}-y\right)^{2} / 2 L \\ \vec{\theta}=\left(\theta_{x}, \theta_{y}\right), \quad \theta_{x}=x_{S} / L, \\ \theta_{y}=x_{S} / L, \quad \theta=\sqrt{\theta_{x}^{2}+\theta_{y}^{2}} .\end{array}\right.$

Actually $\theta$ is the diffraction angle. For the case $\rho_{0}^{2}<<L / k_{0}$, using Eq.(58) the expression for $E_{-1}$ can be rewritten with the accuracy of constant phase in the following form

$$
\begin{aligned}
& E_{-1}=\frac{k_{0} I_{0}}{4 \pi L} W_{0}(t) \int_{-\infty}^{\infty} d \vec{r}_{\perp} \frac{\exp \left[-r_{\perp}^{2} / \rho^{2}\right\rfloor}{1+n_{f}^{2}(0)} \times \\
& \times \sum_{\vec{q}^{2}=q_{0}^{2}} \exp \left[i\left(\vec{q}-\vec{\theta} \cdot k_{0}\right) \cdot \vec{r}_{\perp}\right] \tilde{R}(\vec{q}) .
\end{aligned}
$$

Here $\rho^{2}=\rho_{0}^{2}\left(1+n_{f}^{2}(0)\right)$ and the function depending only on time is introduced (see (59)):

$W_{0}(t) \approx \exp \left[i \frac{\Omega}{2} t\right] \cdot \sin \left(\frac{\Omega}{2} t\right)$.

The random function $\tilde{R}(\vec{q})$ is defined from Eq.(59b).

After integration of Eq.(A.3) with the accuracy of constant phase one can obtain

$$
\begin{aligned}
& E_{-\perp} \cong \frac{k_{0} \rho_{0}^{2} I_{0}}{4 L} W_{0}(t) \times \\
& \times \sum_{\vec{q}^{2}=q_{0}^{2}} \tilde{R}(\vec{q}) \exp \left[-\frac{-k_{0}^{2} \rho^{2}}{4}\left(\vec{\theta}-\vec{q} / k_{0}\right)^{2}\right]
\end{aligned}
$$

Let us take into account that $\vec{\theta} \cdot \vec{q}=\theta \cdot q_{0} \cdot \cos \varphi$, and replace the summarizing on $\vec{q}$ in (A.5) with the integration on $\varphi \in[0,2 \pi]$. Note that the average value of (A.5) on the random sources is equal to zero. In order to obtain the intensity we will use Eq. (59b): 
A.N. Morozovska, V.V. Obukhovsky: Spatial-temporal peculiarities of periodical PILS ...

$$
\left\langle\widetilde{R}(\vec{q}) \widetilde{R}^{*}\left(\vec{q}^{\prime}\right)\right\rangle=\delta\left(\vec{q}^{\prime}-\vec{q}\right)\left\langle\left|\widetilde{R}^{2}\left(q_{0}\right)\right|\right\rangle,
$$

and the integral representation of the modified Bessel function of zero order $B_{0}$ and its asymptotic form:

$$
\begin{aligned}
& B_{0}(x)=\frac{1}{2 \pi} \int_{0}^{2 \pi} d \varphi \exp [x \cos \varphi] \approx \\
& \approx\left\{\begin{array}{l}
\frac{1}{\sqrt{2 \pi|x|}} \exp (|x|), \quad|x|>>1 ; \\
1, \quad|x|<<1 .
\end{array}\right.
\end{aligned}
$$

Using Eqs (A.5) and (A.6), one can get the intensity on the screen as follows:

$$
\begin{aligned}
& I_{-1}(\theta)=R_{0}^{2}\left|W_{0}(t)\right|^{2} \times \\
& \times \exp \left[-\frac{\Delta^{2}}{2}\left(\theta^{2}+\theta_{0}^{2}\right)\right] B_{0}\left(\Delta^{2} \theta_{0} \theta\right) \approx \\
& \approx R_{0}^{2}\left|W_{0}(t)\right|^{2} \frac{\exp \left[-\frac{\Delta^{2}}{2}\left(\theta-\theta_{0}\right)^{2}\right]}{1+\pi \Delta^{2} \theta_{0} \cdot \theta}
\end{aligned}
$$

In Eq. (A.7) the following designation are introduced

$$
R_{0}^{2}=\left\langle\left|\left(1+\alpha_{1}\right) \frac{R_{1} \alpha_{2}-R_{2}}{\alpha_{1}-\alpha_{2}}\right|^{2}\right\rangle\left(\frac{k_{0} \rho_{0}^{2}}{2 L}\right)^{2} \times
$$$$
\times \frac{I_{0}^{3}}{1+\left(\ell-z_{0}\right)^{2} / k_{0}^{2} \rho_{0}^{4}} \frac{\exp \left[2 s_{0} \ell\right]}{s_{0}^{2}+q^{2} / 4 k_{0}^{2}},
$$$$
\theta_{0}=q_{0} / k_{0}, \quad \Delta=k_{0} \rho=\frac{F}{a_{0}},
$$

$$
\rho^{2}=\rho_{0}^{2}\left(1+n_{f}^{2}(0)\right)
$$

The angle brackets mean the averaging on the random sources $R$. 